\title{
Regímenes de Integración Regional: la construcción institucional de los mercados del sur global ${ }^{1}$
}

\author{
Stefano Palestini Céspedes*
}

\begin{abstract}
Resumen
El presente ensayo introduce el concepto de régimen de integración regional como una herramienta analítica para el estudio comparado de los procesos de integración en economías emergentes. A partir de este concepto se analiza el desarrollo histórico de la integración regional centrándose en Sudamérica y el Sudeste Asiático, estableciendo los vínculos estructurales entre integración regional y desarrollo capitalista, para luego describir las características de los regímenes de integración contemporáneos. El artículo finaliza presentando algunas hipótesis desde la economía política acerca de la profundidad de la integración y la naturaleza del cambio en estos regímenes.

Palabras clave: Regímenes de integración - desarrollo capitalista - transnacionalización - economía política.

Abstract

The essay introduces the concept of regional integration regime as an analytical tool for the comparative study of integration processes in emergent economies. Using this concept, the author analyzes in first place the historical path of regional integration in South America and Southeast Asia, establishing the structural relations between regional integration and capitalist development. In second place, the institutional characteristics of contemporary integration regimes are described. The paper concludes advancing some hypotheses -based on political economy-about the depth of the integration and the nature of change within these regional regimes.
\end{abstract}

Keywords: Integration regimes - capitalist development - transnationalization - political economy.

1 La versión inglesa del presente artículo fue presentada en la Cuarta Conferencia de la Red de Graduados en Ciencias Sociales en la Universidad Alexander von Humboldt, Alemania (24-marzo-2012), y es parte de la tesis doctoral del autor acerca de las diversidades de capitalismo regional en economías emergentes. El autor agradece los comentarios, críticas y sugerencias de Visnja Vukov, Lászlo Bruszt, y los dos evaluadores anónimos. Los errores y falencias son responsabilidad exclusiva del autor.

* Sociólogo y Candidato a Doctor en Ciencias Políticas y Sociales, Instituto Universitario Europeo de Florencia. Correo electrónico: stefano.palestini@eui.eu 


\section{INTRODUCCIÓN}

Este artículo busca introducir el análisis de la integración regional desde la economía política y en perspectiva comparada, con el fin de discutir los cambios de contenido que ha tenido el concepto de integración en diversas economías capitalistas. La referencia empírica para analizar estos procesos de cambio son los procesos de integración y cooperación regional en las economías emergentes del sur a través de una perspectiva que se diferencia tanto de los estudios de integración europea que se han centrado exclusivamente en el análisis institucional del Viejo Continente (véanse los enfoques citados en Wiener y Diez, 2004), como de aquellos enfoques que han analizado procesos de integración en otras regiones, pero utilizando Europa como "modelo" empírico y normativo (véanse, por ejemplo: Lenz, 2011; Jetschke, 2009; Welfens et al., 2006; Tèlo, 2007). A diferencia de dichos estudios, en este ensayo se argumenta que los contenidos de la integración varían espacialmente dependiendo de la economía política regional que se esté analizando, y temporalmente dependiendo de la etapa de desarrollo capitalista en la cual esté inmersa. Es por ello que, así como existe una diversidad de formas de capitalismos nacionales, existe también una variedad de formas de capitalismos regionales (Phillips, 2004). La comparación entre regiones distintas no tiene, por tanto, un objetivo normativo -estudiando, por ejemplo, los grados de variación de ciertos casos respecto a un modelo o paradigma de integración-, sino un sentido heurístico, cual es el de identificar los patrones de similitudes y diferencias en una perspectiva temporalhistórica y espacial-geográfica.

El ensayo se concentra en Sudamérica y el sudeste asiático, dos regiones de lo que se ha venido conociendo como el sur global -es decir, las economías del hemisferio sur con altos niveles de inserción en la economía global-. Para efectuar el análisis comparativo de estas regiones se utiliza el concepto de régimen de integración regional que será definido y discutido en la primera parte del ensayo. Mi argumento es que dichos regímenes se han transformado históricamente en directa relación a los cambios de los modos de acumulación capitalista acontecidos en ambas regiones. En otras palabras, integración regional y desarrollo del capitalismo están estructuralmente vinculados. En la segunda parte del ensayo se ilustra esta evolución histórica. En la tercera parte se abordan las características de los regímenes de integración regional contemporáneos durante la primera década del siglo XXI. En la cuarta parte y final presento tres perspectivas teóricas de la economía política internacional que interpretan la naturaleza de las instituciones regionales contemporáneas. Las preguntas que están en juego para estas perspectivas antagónicas son: cuál es el significado y profundidad de la integración regional en el momento actual, y hasta qué punto existe una discontinuidad con las etapas anteriores.

\section{REGÍMENES DE INTEGRACIÓN REGIONAL: HACIA UNA DEFINICIÓN OPERATIVA DEL CONCEPTO}

La noción de régimen ha estado siempre presente en el vocabulario de las ciencias sociales sin gozar, no obstante, de una definición precisa. En el campo de las relaciones internacionales, tal definición vendría de la mano de los trabajos de Stephen Krasner (1982) 
y John Ruggie (1982), quienes comenzarían a utilizar el concepto de régimen como una herramienta teórica para el análisis de la economía política internacional.

Para Krasner un régimen es un conjunto de principios, normas, reglas y procedimientos que permiten la coordinación de las expectativas de los actores sociales en un espacio social determinado, que en el caso de Krasner y sus colegas es el internacional ${ }^{2}$. De acuerdo a Krasner, los principios son creencias acerca de los hechos y sus relaciones causales así como acerca de la rectitud de las acciones, es decir, creencias acerca lo que es cierto y lo que es justo. Las normas son pautas de comportamiento definidas en términos de derechos y obligaciones. Las reglas son prescripciones o proscripciones que regulan el comportamiento. Finalmente los procedimientos son prácticas institucionalizadas acerca de cómo tomar e implementar decisiones (Krasner, 1982).

En su definición, Krasner enfatizó la distinción entre, por una parte, principios y normas, y, por la otra, reglas y procedimientos. En efecto, entre los cuatro elementos hay una jerarquía de lo abstracto a lo concreto que va desde las creencias tácitas acerca de lo que es verdadero y justo hasta las formas concretas de acción. Los principios gobiernan las normas que a su vez gobiernan las reglas que definen los procedimientos. Sin embargo, la distinción va más allá del terreno puramente analítico, pues se relaciona con los tipos empíricos de cambio social. El cambio en reglas y procedimientos implica un cambio en el régimen, mientras el cambio de principios y normas implica un cambio de régimen.

La distinción es extremadamente relevante para el análisis comparativo de regímenes internacionales y, específicamente en el caso de este ensayo, regímenes regionales. En efecto, buena parte de la literatura acerca del cambio institucional en ciencias políticas y sociología ha tendido a enfocarse en cambios de reglas y procedimientos, y sólo en pocos casos cambios normativos y de principios. El debate contemporáneo entre quienes abogan por una teoría del cambio gradual y una teoría del cambio abrupto o puntuado (véase Streeck y Thelen, 2009) se ha movido en general en el ámbito de las transformaciones a nivel de reglas y procedimientos. Como indica el propio Krasner, la identificación de cambios normativos y de principios no es una tarea fácil para el análisis empírico, pues tal juicio no puede basarse en observaciones de comportamiento sino a través de la reconstrucción interpretativa de la intencionalidad de dichos comportamientos. De ahí que los enfoques del cambio institucional basados en una teoría de la acción racional están menos equipados teóricamente para tal reconstrucción que, por ejemplo, los enfoques constructivistas y sociológicos o aquellos basados en el institucionalismo histórico (para una revisión de las teorías acerca de los regímenes internacionales y sus distintas variantes liberales, racionales y constructivistas, véase Hasenclever et al., 1997).

En el esquema analítico de John Ruggie, reglas y procedimientos constituyen la forma de un régimen, mientras principios y normas son su contenido. Para Ruggie, el contenido de un régimen dice relación con el fin social legítimo del régimen. En mi interpretación, con

2 Véase el número especial de International Organization, vol. 36, número 2, año 1982. 
este concepto (legitimate social purpose) Ruggie no se refiere a la finalidad teleológica del funcionalismo, según el cual un régimen podría entenderse como un sistema social orientado por el cumplimiento de una función. Para Ruggie la finalidad o propósito de un régimen depende de la legitimidad que dicho propósito represente para los actores que participan de él y está sujeto a las variaciones en la distribución de poder y autoridad de dichos actores, emparentándose así a la concepción weberiana de finalidad.

A través de esta distinción, Ruggie analiza los regímenes internacionales del dinero y el comercio posteriores a la Segunda Guerra Mundial. Su argumento esencialmente es que en el período de entreguerras ocurre una transformación de los principios del liberalismo ortodoxo que caracterizó al largo siglo XIX. Dicha transformación implicó la emergencia de un nuevo régimen internacional al cual, parafraseando a Karl Polanyi, denomina liberalismo arraigado (embedded liberalism). Mientras el contenido del liberalismo ortodoxo consistía en la creciente expansión de los mercados, con la consiguiente mercantilización (commodification) de las esferas de la vida social, los regímenes del liberalismo arraigado de la posguerra prescribirían la acción de las instituciones políticas con el fin de contener la dislocación social y económica producida por la creciente mercantilización (Ruggie, 1982).

Basándose en el concepto de régimen de Krasner y Ruggie, Lászlo Bruszt y Gerald McDermott han comenzado a utilizar el concepto de régimen transnacional de integración en el análisis comparativo de los procesos de transnacionalización en diversas regiones y sus efectos sobre las instituciones y los actores domésticos (Bruszt y Holzhacker, 2009; Bruszt y McDermott, 2011). Los regímenes transnacionales de integración crean y gobiernan las normas y reglas de la interacción económica en un espacio transnacional específico. Siguiendo a Ruggie, dichos regímenes varían dependiendo de sus fines y el uso del poder. Bruszt y McDermott operacionalizan fines como metas institucionales y poder, como el tipo de estructuras de gobernanza (governance structure) que los regímenes comprenden (Bruszt y McDermott, 2011: 2).

El concepto de régimen transnacional de integración es muy útil para el análisis comparativo de las instituciones transnacionales y sus efectos en diversas regiones así como en diversos sectores (políticos, económicos, de políticas públicas, etc.), abriendo así una promisoria agenda de investigación. Sin embargo, en mi opinión la operacionalización del concepto que estos autores realizan pierde de vista la distinción entre forma y contenido del régimen como fuera expuesta por Krasner y Ruggie en los ochenta. En efecto, Bruszt y McDermott operacionalizan fines como metas institucionales, las que varían de acuerdo a dos dimensiones: alcance y profundidad. En la primera dimensión las instituciones pueden ser "estrechas" si se focalizan exclusivamente en reglas económicas y de comercio, o "extensas" si regulan ámbitos sociales y políticos. La segunda dimensión, en tanto, profundidad, puede ser "superficial" si las instituciones se enfocan exclusivamente en cambios de políticas, o "profunda" si enfatizan la construcción de capacidades administrativas, de seguimiento y de enforcement. A través de esta operacionalización, los autores pueden identificar variación en los procedimientos y reglas que, en el caso de su estudio, la Unión Europea y el NAFTA utilizan en la regulación de mercados transnacionales. Sin embargo, en mi opinión, no logran 
capturar cambios en el contenido, es decir, en los principios y normas que gobiernan a la Unión Europea y a NAFTA respectivamente en cuanto regímenes de integración (Bruszt y McDermott, 2011).

Para ir más allá de la comparación formal de regímenes regionales o transnacionales y dar cuenta del contenido normativo, es necesario, en mi opinión, analizar empíricamente el significado que ha adquirido la integración para cada régimen. En efecto, el concepto de integración ha variado histórica y espacialmente de un régimen a otro, pudiendo identificarse al menos cuatro conceptos analíticamente distintos y con raíces en distintas literaturas: el concepto económico de integración como aumento del intercambio de bienes, servicios y capital (Balassa, 1973; Bhagwati, 2004); el concepto regulativo de integración como progresiva armonización de procedimientos y reglas (Stone Sweet y Sandholtz, 1997; Bruszt y Holzhacker, 2009); el concepto sociológico de integración como creciente cohesión y decreciente conflicto entre grupos sociales y organizaciones (Durkheim, 1997 [1893]; Lockwood, 1964); y el concepto político de integración como creciente delegación de la autoridad y actividades políticas desde los centros nacionales a un centro supranacional (Haas y Schmitter, 1964; Schmitter y Malamud, 2007).

El análisis de los regímenes regionales o trasnacionales de integración debe, en mi opinión, prestar atención a la manera que la integración ha sido normativamente definida, así como a las reglas y procedimientos que la han concretizado. En la segunda parte de este artículo se describen los cambios de contenido que han tenido los regímenes regionales en Sudamérica y el sudeste asiático. El argumento, de algún modo materialista, es que dichos cambios normativos han ido de modo paralelo a las transformaciones en los modos de acumulación que dichas regiones han experimentado. Este análisis podría efectuarse con otras regiones y otros procesos de integración. Se ha elegido a estas dos regiones pues poseen paralelos interesantes en sus dinámicas institucionales tanto desde una perspectiva de mediano plazo como en cuanto a sus características contemporáneas. Además, poseen similares características de desarrollo económico y desarrollo humano, ambas son regiones periféricas en la economía global y ambas iniciaron sus procesos de industrialización e integración regional de modo contemporáneo.

\section{REGÍMENES DE INTEGRACIÓN REGIONAL EN PERSPECTIVA HISTÓRICA}

La integración regional en Europa y en las economías periféricas avanzó, durante la segunda mitad del siglo XX, en forma paralela a la expansión de los mercados, la acumulación del capital y la mercantilización de las esferas sociales, principales dimensiones del desarrollo capitalista. Por cierto, las fases del desarrollo del capitalismo no constituyen la historia completa de la integración regional pues en efecto otros procesos como la construcción de comunidades de seguridad y los procesos de democratización han sido importantes vectores de la integración regional, particularmente en el sudeste asiático y en Sudamérica. Sin embargo, la tesis que quiero defender en esta sección es que para comprender las transformaciones formales, pero especialmente de contenido de los regímenes regionales en el sur global, es imprescindible ponerlos en el contexto de sus trayectorias de desarrollo 
en tanto regiones periféricas de la economía capitalista global. De lo contrario se corre el riesgo de que la integración regional aparezca como un epifenómeno institucional aislado y, como se diría desde la tradición marxista, indeterminado y ahistórico ${ }^{3}$.

\subsection{Industrialización por sustitución de importaciones y regionalismo políticamente conducido}

La "gran transformación" de las economías sudamericanas y del sudeste asiático, es decir, el momento en que ellas se convierten en economías capitalistas de mercado, fue un duradero y fragmentado proceso que cubrió, con avances y retrocesos, diversos momentos de la historia moderna de estas regiones. Sin embargo, en el caso de las economías mayores de Sudamérica -Brasil, Argentina, Chile, Colombia, Venezuela y habría que agregar, por el norte, a México- es posible identificar la década del treinta como el inicio de un proceso de industrialización acelerada con miras a desencadenar el proceso de acumulación capitalista después de los catastróficos efectos de la Gran Depresión sobre la demanda de materias primas por parte de las economías centrales. Dicho proceso de acumulación, así como su estrategia de desarrollo, han sido normalmente conocidos por la historiografía económica como Industrialización por Sustitución de Importaciones (ISI), si bien recientemente economistas e historiadores económicos han preferido utilizar el rótulo de "industrialización acelerada" (Cárdenas et al., 2000). Este proceso se extenderá con variaciones menores entre países hasta la década del setenta, teniendo su mayor auge en las décadas del cincuenta y sesenta, período en el cual las estructuras productivas de las economías mayores, seguidas en un efecto de eco por las economías más pequeñas, modernizaron sus estructuras productivas generando industrias de bienes de consumo y, en algunos casos como Brasil, Argentina y Chile, industrias de bienes de capital, con miras a transformar la inserción de las economías sudamericanas en la economía internacional. Durante estas décadas se iniciará, como veremos enseguida, la moderna historia de la integración regional en Sudamérica y Latinoamérica.

Girando rumbo al este, los procesos de industrialización de las economías del sudeste asiático se inician en la década de los cincuenta y sesenta, con un retardo de dos décadas respecto a las economías sudamericanas. Dentro de las economías del sudeste asiático es necesario hacer una distinción entre aquellas del primer anillo de industrialización (Singapur y, eventualmente, Malasia), el segundo anillo (Indonesia, Tailandia y Filipinas) y el resto de las economías de la península indochina completamente periféricas y con un patrón de industrialización prácticamente inexistente.

Al igual que los Ilamados tigres asiáticos o nuevas economías industrializadas (Hong-Kong, Corea y Taiwán), Singapur y en menor medida Malasia generaron un modelo de acumulación capitalista sobre la base de la substitución de importaciones acoplado con una estrategia

3 En esto sigo las premisas de Wolfgang Streeck acerca de la necesidad de recuperar para la economía política una noción no teleológica de desarrollo y evolución, de la cual buena parte de los estudios comparados carece (Streeck, 2009: véase la entrevista publicada en http://estudiosdelaeconomia.wordpress.com/2011/04/14/ entrevista-a-wolfgang-streeck/). 
de fomento de las exportaciones. Por un par de décadas los economistas que analizaron el Ilamado milagro asiático de estas economías sostuvieron la tesis -con enormes repercusiones para otras regiones en desarrollo como Sudamérica, África Subsahariana, o la Europa del Este- de que dicho modelo de desarrollo hacia afuera era conducido exclusivamente por el mercado, es decir, basado en un modelo de liberalización y capital transnacional. Hoy ha sido establecido que, muy por el contrario, fue un proceso activamente conducido por el Estado y por políticas industriales activas y selectivas (Di Maio, 2011; Gereffi y Wyman, 1990). Las economías del segundo anillo -Indonesia, Filipinas y Tailandia-, en cambio, siguieron un modelo similar al sudamericano, es decir, un modelo de industrialización por substitución de importaciones "hacia adentro", reforzado por el nacionalismo económico de estas economías poscoloniales que, no obstante, continuaban siendo dependientes del capital extranjero de las ex metrópolis para la importación de bienes de capital ${ }^{4}$.

En la década del sesenta, y en el marco de estos procesos de ISI, surgen sendos regímenes de integración regional. En 1960 se crea, a través del Tratado de Montevideo, la Asociación Latinoamericana de Libre Comercio (ALALC) integrada por Argentina, Brasil, Chile, Paraguay y Perú más México. En 1967, en tanto, se crea, por medio de la Declaración de Bangkok, la Asociación de Naciones del Sudeste Asiático (ASEAN) integrada por Indonesia, Filipinas, Malasia, Singapur y Tailandia. En cuanto régimen de integración regional, ALALC estuvo íntimamente ligada a la ISI. En efecto, ambas fueron proyectos diseñados por el grupo de economistas y sociólogos de la Comisión Económica para América Latina (CEPAL). A través de la liberalización de las tarifas aduaneras y del fomento de la complementación productiva entre las economías nacionales, ALALC perseguía la construcción de un mercado regional para los bienes de consumo producidos por las nuevas industrias nacionales; dicho mercado regional era imprescindible para sostener el proceso de industrialización, debido a las restricciones de tamaño de los mercados nacionales. El mercado regional se concebía, además, como una alternativa al mercado internacional en el cual los bienes latinoamericanos no eran competitivos debido a su alto costo de producción (Philips, 2004). En su contenido, ALALC se puede entender, por tanto, como la ampliación a nivel continental del proceso de industrialización por substitución de importaciones, bajo los principios de cooperación regional. Formalmente, en tanto, se caracterizó por el uso de la liberalización aduanera y la complementariedad productiva como instrumentos de integración.

ASEAN, por su parte, nació no a pesar, sino a causa de los agudos conflictos bélicos entre las naciones de la región con el fin de generar paz y estabilidad, fortalecer los principios de la ley y acelerar el crecimiento económico y la industrialización de la región. Los líderes de ASEAN comprendieron que el éxito económico de sus países era la mejor garantía para la paz: la República Popular China creada en 1949 había desatado los conflictos este/oeste en Asia; a ello había que sumar la partición de Vietnam y la sucesión de conflictos en Indochina.

4 Un ejemplo de este modelo de acumulación fue la llamada indeonesianisation de la economía de Indonesia en la década del cincuenta, en la que se combinó un fuerte proteccionismo y fomento de la economía por parte del Estado que incluyó la creación de bancos y firmas estatales de modo de reducir la dependencia del capital holandés. 
Asimismo, entendieron que la modernización económica y la prosperidad, más que la naturaleza democrática de sus sistemas políticos, constituían la mejor fuerza magnética para acercar a sus vecinos comunistas a una afiliación regional común. En su contenido ASEAN es similar a las Comunidades Europeas de las posguerra, basándose en los principios de estabilidad, seguridad regional, cooperación y generación de comunidad (Kühnhardt, 2010; Gilbert, 2012). En su forma, ASEAN utilizó los instrumentos de la integración económica y el diálogo intrarregional.

Las normas de no-interferencia y respeto a la autonomía nacional conocidos como "ASEAN way" del cual derivan mecanismos de toma de decisiones basados en el consenso y una baja institucionalización, permitió a ASEAN avanzar según el timing de los conflictos de Indochina, dando pasos graduales en términos de modernización económica y de construcción de comunidad (Kühnhardt, 2010; Beeson, 2009; Alexander, 2011).

Sin embargo, a finales de los años setenta los indicadores de integración económica en el sudeste asiático comienzan a mostrar un paulatino estancamiento permaneciendo relevantes exclusivamente los flujos comerciales bilaterales entre Indonesia y Malasia, y Singapur y Malasia. En los ochenta, ASEAN no jugaría prácticamente ningún rol en cuanto a la integración económica y a la industrialización, permaneciendo de manera exclusiva como una organización con objetivos de seguridad, mientras el desarrollo económico sería conducido únicamente por políticas nacionales de carácter proteccionista. Por el mismo período en Sudamérica, ALALC inicia un agónico periplo hasta desaparecer completamente en 1980. La crisis de ASEAN y ALALC en cuanto regímenes de integración regional se explica por el agotamiento y crisis de la industrialización por substitución de importaciones en ambas regiones. En otras palabras, y utilizando los términos de la Escuela de la Regulación, los procesos de integración se vieron afectados por una crisis del modelo de acumulación capitalista (Boyer, 2007). En efecto, el aumento del precio de las materias primas en el mercado internacional incentivó el retorno a un modelo de acumulación extensivo basado en la exportación de dichos bienes, reforzando un tipo de inserción internacional dependiente y periférico. A estas condiciones externas o estructurales hay que agregar los factores internos -coaliciones de clases y decisiones políticas- que llevarían a las economías sudamericanas, así como del sudeste asiático (con la excepción de Singapur y Malasia que seguirían el modelo de los tigres asiáticos), por rutas diversas -pero igualmente dependientes- de desarrollo (Cardoso y Faletto, 1969; Cardoso, 2009; véase Bruszt y Greskovits en el número 26 de la Revista de Sociología). Estas economías volvieron a ser competidores entre ellas en los mercados de materias primas, socavando los principios de complementariedad y cooperación sobre los que se basaba la integración en ambas regiones.

ALALC y ASEAN comparten características de forma y contenido, que las hacen pertenecer a un mismo modelo de integración regional políticamente conducidos. Por cierto, los regímenes que surgirán en los años ochenta y noventa, así como los regímenes contemporáneos, poseen, siguiendo a Ruggie, fines sociales legítimos, y por ende son ellos también de alguna manera proyectos políticos. Sin embargo, lo que une a ALALC y a ASEAN de los años setenta, así como las Comunidades Europeas -si quisiésemos extender el análisis a Europa-es, por una 
parte, la definición social y política que le dan a la integración regional y, por otro, el lugar que le asignan a las instituciones políticas en la conducción de dicho proceso.

La conducción política del proceso de integración regional de estos proyectos se ve reflejada en el texto del Secretario Ejecutivo de ALALC en 1969:

\begin{abstract}
"La integración económica latinoamericana debería ser vista también como una respuesta de nuestros países al desafío que ha sido planteado desde el exterior, en vista al hecho de que los países del mundo están tendiendo a organizarse ellos mismos en grandes comunidades geoeconómicas, conscientes de su destino común y de la necesidad de defender sus intereses comunes. Desde sus orígenes ALALC ha compartido esta concepción y en más de una ocasión se ha enfatizado que su nacimiento fue un reflejo en nuestro continente del movimiento europeo que se cristalizó en 1957 con la creación de la Comunidad Económica Europea" (citado en Philips, 2004).
\end{abstract}

\title{
2.2. Neoliberalismo y regionalismo abierto
}

No tengo ninguna duda que el siglo que viene será ineludiblemente marcado por el paso hacia una economía cada vez más globalizada y con una tendencia clara hacia el regionalismo abierto.

Presidente Carlos Saúl Menem, 1999

La década de 1980 sería el inicio tanto de un nuevo modo de acumulación como de una nueva ola de integración regional en ambas regiones. La crisis de la deuda externa se inició en la economía mexicana para luego extenderse por el resto de las economías latinoamericanas y otras economías periféricas, ya fuertemente debilitadas por la inflación y el estancamiento económico, fenómeno conocido como stagflation. El diagnóstico de las instituciones financieras internacionales fue que las economías periféricas estaban sufriendo las consecuencias negativas del proteccionismo, la falta de competitividad y una desproporcionada deuda pública. La medicina, en tanto, fueron los programas de ajuste estructural dirigidos por el Fondo Monetario Internacional, los cuales iniciarían una nueva etapa en el desarrollo capitalista de Sudamérica, el sudeste asiático, el Asia Subsahariana y la Europa del Este (véase el artículo de Bruszt y Greskovits, 2011, en Revista de Sociología, 26).

De modo paralelo, una nueva ola de regionalismo, denominado nuevo regionalismo o regionalismo abierto, empezaría a difundirse de norte a sur y de este a oeste. Probablemente, lo único en común entre los viejos regímenes regionales políticamente conducidos (ALALC y el ASEAN de los años setenta) y los nuevos regímenes neoliberales sea la palabra región. Tal como los geógrafos económicos y el constructivismo en general han argumentado, una "región" es una construcción sociopolítica, y las nuevas regiones serían delineadas en el marco de un mundo completamente distinto al de la guerra fría, y, lo más importante para nuestro argumento, bajo un modo de acumulación y una ideología de desarrollo diferentes. Los tratados regionales de comercio (regional trade agreements), como empezarían a ser conocidos los nuevos regímenes, fueron concebidos como un instrumento para incrementar la capacidad de las economías en desarrollo de atraer Inversión Extranjera Directa (IED) que, 
de acuerdo al pensamiento neoliberal, era el principal vector de crecimiento económico y de inserción internacional. En su dimensión de política externa, el neoliberalismo -que informaba la agenda de las instituciones financieras internacionales- sostenía que la IED era fundamental para paliar la carencia de activos financieros y de tecnología imprescindibles para echar a andar las economías estancadas de las regiones en desarrollo. La fase del capitalismo transnacional -caracterizada por el acelerado proceso de transnacionalización de los mercados productivos y de capitales- se iniciaría tanto en Sudamérica como en el sudeste asiático a inicios de los años ochenta, desatada por la liberalización de los mercados financieros internacionales y por la llegada masiva de empresas transnacionales con matrices en las economías centrales y Japón. Las empresas transnacionales japonesas, estadounidenses y europeas iniciaron un viaje a través de las regiones en desarrollo siguiendo el modelo que el economista japonés Kaname Akamatsu había llamado flying geese (el vuelo de los gansos). Según este modelo, las empresas transnacionales japonesas liderarían una cadena de producción transnacional en la que los distintos eslabones estarían integrados jerárquicamente por economías menos avanzadas aprovechando las ventajas comparativas de cada economía. Durante los noventa a través de este modelo se aumentarían los flujos de IED en regiones en desarrollo de 159,3 miles de millones de dólares a 400,5 miles de millones de dólares, transformando la configuración de la economía global y la división internacional del trabajo (Dicken, 2007).

Los tratados de comercio regional serían concebidos, por una parte, como instrumentos fundamentales para atraer las inversiones de dichas empresas e integrarse, a través de cadenas transnacionales, a la economía global. Por otra parte, ellos ofrecían la posibilidad de una liberalización gradual (el mercado regional como un paso previo al mercado global) que permitiría mitigar los efectos de ajuste, así como la oposición política a la liberalización de los sectores del capital doméstico y del trabajo (Bowles, 2002).

En 1991 se funda, bajo el Tratado de Asunción, el Mercado Común del Sur (MERCOSUR ${ }^{5}$ ) integrándolo Argentina, Brasil, Paraguay y Uruguay, y luego ampliándose, con la asociación de Bolivia y Chile, en 1996. EI MERCOSUR de los noventa es el más claro ejemplo de un régimen regional de integración neoliberal, en el que prácticamente todos los legados del viejo regionalismo de ALALC fueron abandonados. Durante su primera década, la agenda del MERCOSUR se focalizó en la integración comercial con miras a generar una unión aduanera regional y, secundariamente, generar lazos comerciales externos a la región. Un año después a la creación del MERCOSUR se funda el Área de Libre Comercio de ASEAN (AFTA) con el fin de integrar comercialmente las economías del sudeste asiático y de atraer las inversiones japonesas en un esfuerzo por competir con las potencias y nuevos países industrializados del noreste asiático: China, Corea, Taiwán y Hong Kong.

5 Si bien en lo que sigue nos focalizaremos en el MERCOSUR y en UNASUR, cabe destacar que la Comunidad Andina de Naciones (CAN) corresponde también a un régimen de integración regional de tipo neoliberal. Fue creada oficialmente por el Acta de Trujillo en 1996 como sucesora del Pacto Andino, régimen políticamente conducido creado en 1969 fuertemente influido por las premisas de la teoría de la dependencia. 
Tanto el MERCOSUR como el AFTA durante sus primeros años pueden considerarse, bajo el nuevo paradigma, como regímenes exitosos a partir de indicadores como integracióncomercial y flujos de IED. No obstante, como veremos en la próxima sección, los éxitos de esa primera década de integración regional neoliberal culminaron con una sucesión de crisis que minaría buena parte de los éxitos acumulados.

TABLA 1

Regímenes de integración regional políticamente conducidos y neoliberales

\begin{tabular}{|c|c|c|c|c|}
\hline $\begin{array}{l}\text { MODO DE } \\
\text { ACUMULACIÓN } \\
\text { CAPITALISTA }\end{array}$ & $\begin{array}{c}\text { RÉGIMEN } \\
\text { REGIONAL DE } \\
\text { INTEGRACIÓN }\end{array}$ & & $\begin{array}{l}\text { CONTENIDO, } \\
\text { PRINCIPIOS Y } \\
\text { NORMAS }\end{array}$ & $\begin{array}{c}\text { FORMA, } \\
\text { PROCEDIMIENTOS } \\
\text { Y REGLAS }\end{array}$ \\
\hline $\begin{array}{l}\text { Intensivo- } \\
\text { industrialización } \\
\text { por sustitución de } \\
\text { importaciones }\end{array}$ & $\begin{array}{l}\text { Políticamente } \\
\text { Conducidos } \\
(1940-1970)\end{array}$ & $\begin{array}{l}\text { ALALC } \\
\text { ASEAN } \\
\text { Comunidades } \\
\text { Europeas }\end{array}$ & $\begin{array}{l}\text {-Integración } \\
\text { como cohesión y } \\
\text { cooperación. } \\
\text {-Región como } \\
\text { unidad política y } \\
\text { económica. } \\
\text {-Fin social } \\
\text { legítimo: } \\
\text { estabilidad } \\
\text { social, desarrollo } \\
\text { económico y } \\
\text { seguridad. }\end{array}$ & $\begin{array}{l}\text {-Liberalización } \\
\text { aduanera } \\
\text { más políticas } \\
\text { industriales de } \\
\text { complementación } \\
\text { productiva. } \\
\text {-Foros de } \\
\text { deliberación } \\
\text { política. }\end{array}$ \\
\hline $\begin{array}{l}\text { Extensivo-orientado } \\
\text { a las exportaciones }\end{array}$ & $\begin{array}{l}\text { Neoliberales } \\
(1980-2000)\end{array}$ & $\begin{array}{l}\text { AFTA } \\
\text { MERCOSUR } \\
\text { CAN } \\
\text { APEC } \\
\text { SADC }\end{array}$ & $\begin{array}{l}\text {-Integración como } \\
\text { intercambio de } \\
\text { bienes, servicios y } \\
\text { capital. } \\
\text {-Región como } \\
\text { mercado y paso } \\
\text { previo al mercado } \\
\text { internacional. } \\
\text {-Fin social } \\
\text { legítimo: } \\
\text { estimulación del } \\
\text { crecimiento y la } \\
\text { competitividad. }\end{array}$ & $\begin{array}{l}\text {-Fomento de los } \\
\text { flujos de IED. } \\
\text {-Eliminación de } \\
\text { barreras aduaneras } \\
\text { y aranceles } \\
\text { externos. } \\
\text {-Desregulación } \\
\text { doméstica como } \\
\text { instrumento } \\
\text { de reducción } \\
\text { de costos de } \\
\text { transacción para el } \\
\text { capital doméstico y } \\
\text { externo. }\end{array}$ \\
\hline
\end{tabular}


Como se puede apreciar en la Tabla 1, hay diferencias fundamentales entre los regímenes de integración políticamente conducidos y neoliberales, tanto de contenido como de forma. Como se dijo en la primera parte del ensayo, de acuerdo a los teóricos de los regímenes internacionales son los cambios de contenido los que permiten identificar el paso de un régimen a otro. Los regímenes políticamente conducidos entendieron "integración" como el fortalecimiento y la ampliación de los vínculos entre sociedades nacionales con el fin socialmente legítimo de asegurar la estabilidad política, la paz y generar desarrollo económico y social. A su vez, la "región" se entendió como una unidad social y política, a la vez que económica. La conciencia del origen y destino común de los países latinoamericanos y de ocupar una misma posición en la estructura del capitalismo internacional -en el pensamiento de Prebisch- están a la base de los principios por los cuales se fundó ALALC (CEPAL, 1959; para una revisión de las ideas de Prebisch y, particularmente, la integración regional, véase Ocampo, 2001). De un modo similar, los líderes de los cinco países fundadores de ASEAN compartieron la visión de que la única manera de sobrevivir en su calidad de pequeños países poscoloniales rodeados por potencias con intereses expansionistas y territoriales, y abrumados por conflictos étnicos y culturales, era fundando una comunidad políticoeconómica con base regional. Es este significado fuerte de región y de integración lo que permite hablar de ASEAN y ALALC como regímenes políticamente conducidos.

El MERCOSUR y el AFTA, en tanto, se fundan en principios diversos. Ambos regímenes comparten una noción débil de integración elaborada principalmente por la economía y fuertemente influida por los modelos del mercado común europeo y del NAFTA. La integración es entendida como el aumento de los intercambios entre países de la región de bienes, servicios y capital. En esta definición predomina la visión económica de integración, según la cual la integración es más perfecta en la medida en que se eliminen las barreras y se robustezcan los lazos comerciales entre naciones (Nesadurai, 2009; Phillips, 2003, 2004; Dieter, 2009). Lo que en los regímenes políticamente conducidos era forma, en los regímenes neoliberales es reificado y convertido en contenido. Del mismo modo, el concepto de región es equiparado con mercado regional perdiendo las dimensiones políticas y sociales del concepto. El mercado regional se entiende, además, como el trampolín (the stepping stone) al mercado internacional, que en la visión neoliberal de la economía sería el único mercado posible y deseable.

Estas diferencias de contenido se traducen, por cierto, en diferencias formales. Los principales instrumentos de integración económica en el ASEAN de 1970 y ALALC eran la liberalización aduanera con miras a generar un área regional de comercio, acompañada con políticas industriales de complementariedad productiva. Este segundo elemento desaparece completamente en el MERCOSUR y en el AFTA; la complementariedad productiva será entendida como resultado natural de las ventajas comparativas de cada economía, y de las estrategias de inversión de empresas transnacionales. Cabe destacar que los sectores de mayor complementariedad productiva -medida como intercambio de bienes intermedios y comercio interindustrial- en Sudamérica y el sudeste asiático son, sin embargo, producto de las viejas políticas industriales de los años setenta y ochenta como lo demuestran los casos de la industria automotriz en Sudamérica y electrónica en el sudeste asiático. Los 
regímenes neoliberales además de rebajar las barreras aduaneras intrarregionales perseguían la reducción o al menos la armonización de las barreras arancelarias externas, característica esencial de un regionalismo abierto, rótulo acuñado, paradójicamente, por la CEPAL, otrora artífice del regionalismo políticamente conducido (cfr. Vázquez, 2011). Finalmente, como hace notar Diana Tussie (2009), los regímenes de integración neoliberal incluyen una agenda de desregulación doméstica orientada a reducir el rol del Estado en la esfera económica con miras a producir ganancias de eficiencia. Estas políticas de desregulación tienen el objetivo de reducir los costos de transacción de las empresas comprometidas en el comercio interregional e internacional.

\section{CRISIS FINANCIERAS Y LA TRANSFORMACIÓN DE LOS CONTENIDOS DE LA INTEGRACIÓN EN EL SUR GLOBAL}

La década de 1990 finalizó con una sucesión de crisis de alcance nacional y regional y una crisis global: la así llamada crisis financiera asiática que se inició en 1997 en Tailandia, para luego extenderse por todo el sudeste y el este de Asia, África y Latinoamérica, con efectos menores en las economías centrales de Europa y Estados Unidos. Diez años más tarde ocurriría la más profunda crisis financiera global desde la Gran Depresión, con serios efectos tanto en las economías centrales como periféricas, para luego culminar con la actual crisis de la deuda soberana que afecta principalmente a la Unión Europea. Estas crisis han tenido, por cierto, causas y mecanismos de contagio diversos, sin embargo todas ellas tuvieron un alcance transnacional, se han difundido a través de redes transnacionales y han tenido como principales protagonistas mercados transnacionales. Además, estas crisis han puesto en evidencia los efectos de veinte años de políticas neoliberales en el debilitamiento de las capacidades de gobernanza y regulación a distintos niveles, ciertamente, nacional, pero también a nivel regional y global (para una opinión contraria véase Braithwaite, 2008).

Es difícil decir si estas crisis representan una crisis mayor del modo de acumulación que ha caracterizado la fase transnacional del capitalismo. Las opiniones académicas en las disciplinas de la economía, la economía política y la sociología económica están divididas. Sin duda la salida de la crisis financiera global del 2008, así como el modo en el cual la Unión Europea emergerá de la crisis de la deuda soberana, serán elementos fundamentales para juzgar acerca de la transformación tanto de la naturaleza del capitalismo contemporáneo como de sus modos de regulación a nivel nacional, regional y global.

En esta sección quiero argumentar que a nivel de los regímenes de integración regional en el sur global ha habido importantes transformaciones, no sólo de forma, sino de contenido. En primer término describiré dichas transformaciones en Sudamérica y el Sudeste Asiático para luego presentar tres perspectivas acerca de la naturaleza de tales transformaciones y los eventuales cambios de regímenes que ellas representan.

La crisis asiática de 1997-1998 marca un punto de inflexión en los indicadores de integración económica tanto en Sudamérica como en el sudeste asiático. Como Alberta Sbragia (2008) ha subrayado, el sorprendente giro de las economías asiáticas desde una integración 
puramente económica, en los 90, a la cooperación regional, en los 2000, fue gatillado por la percepción política de que el manejo de las catastróficas consecuencias de la crisis asiática por parte de las instituciones financieras internacionales era completamente inadecuado. La crisis asiática tuvo un efecto regresivo sobre los niveles de integración comercial y los flujos de IED. En el caso de Sudamérica, y particularmente del MERCOSUR, el efecto de la crisis asiática fue redoblado por la crisis y default de la economía argentina el 2001.

Como consecuencia de dichas crisis, el MERCOSUR fue relanzado el 2004 con el propósito de aumentar su alcance más allá de la integración comercial hacia las áreas de la integración productiva, la coordinación macroeconómica, la integración social y cultural. Este relanzamiento se realizó en el marco de lo que se llamó una agenda por un MERCOSUR político, agenda defendida principalmente por Venezuela, país recientemente admitido al grupo de los miembros plenos del bloque ${ }^{6}$. Dicho MERCOSUR político fue apoyado por los gobiernos de izquierda de Brasil, Argentina y Uruguay. Ese mismo año fue creado el Fondo para la Convergencia Estructural del MERCOSUR (FOCEM) con el fin de crear un mecanismo de redistribución de alcance regional con miras a superar las asimetrías entre las economías pequeñas y las grandes economías del Cono Sur. El FOCEM funciona sobre la base de un sistema de contribuciones proporcional al tamaño de las economías siendo Brasil el principal contribuyente, y Paraguay y Uruguay los principales beneficiarios. El año 2005 se aprobó la creación del Parlamento del MERCOSUR, cuerpo compuesto por 18 delegados de los países miembros con el propósito de paliar el déficit de legitimación democrática que caracterizó el régimen durante los noventa, y con miras a la progresiva construcción de una institucionalidad supranacional.

El año 2000, en la Declaración de Brasilia durante un simbólico primer encuentro de presidentes sudamericanos organizado por el entonces Presidente Fernando Henrique Cardoso se lanza la Iniciativa para la Integración Regional de la Infraestructura de Sudamérica (IIRSA). IIRSA fue planteada como la construcción de la "espina dorsal" del continente y refleja lo que para Cardoso eran los pilares básicos de la integración regional: integración económica, infraestructura física e integración energética (Malamud, 2005). La iniciativa tomaría un cariz menos técnico y más de desarrollo social bajo la administración del Presidente Luiz Inácio Lula Da Silva y convergería con la creación de una Comunidad Sudamericana de Naciones. En efecto, el año 2004 se establecen los tres componentes básicos de la dicha comunidad: la concertación y coordinación de las políticas exteriores, con el fin de afirmar a Sudamérica como grupo regional en las relaciones internacionales; la convergencia de la Comunidad Andina de Naciones (CAN) con el MERCOSUR en un único espacio regional de libre comercio; y la integración energética y de infraestructura bajo IIRSA. Estos pilares darían origen, el año 2007, a la Unión de Naciones Sudamericanas (UNASUR) (Sanahuja, 2011).

6 Al momento de finalizar la redacción de este artículo la incorporación formal de Venezuela fue finalmente ratificada por los presidentes de Argentina, Brasil y Uruguay. Paraguay -país contrario a la incorporación de Venezuela- se encuentra temporalmente suspendido de MERCOSUR. 
Como una directa reacción a la crisis financiera asiática, ASEAN inició, en tanto, un proceso de cooperación regional con otras economías asiáticas, y de manera específica con las potencias del noreste -China, Corea y Japón- fundando el régimen regional de ASEAN +3, el cual ha llegado a convertirse en la más influyente organización regional poscrisis superando, entre otras organizaciones, al Foro de Cooperación Asia-Pacífico (APEC), liderado desde su creación a finales de 1980 por Estados Unidos y que corresponde a lo que se ha denominado en este artículo como un régimen neoliberal de integración. ASEAN y sus mecanismos de toma de decisiones han sido integrados y modelados por ASEAN +3 , dando a las economías del sudeste asiático un rol de eje y conductor (hub and drive) del proceso de integración regional (Ba, 2009; Rajan y Rongala, 2008).

ASEAN+3 incluye diferentes dimensiones de cooperación económica y política desde las áreas de integración física, hasta cooperación en materia de seguridad y derechos humanos (Nesadurai, 2009). En materia de cooperación económica, por ejemplo, ASEAN+3 ha generado mecanismos de cooperación financiera a escala regional, lo cual representa una innovación institucional no solo para el este asiático sino para las regiones en desarrollo en general. La iniciativa Chiang Mai surgió con posterioridad a la crisis financiera asiática como un intento por institucionalizar una serie de acuerdos de swaps, es decir, intercambios a tasas preferenciales de moneda local por divisas (principalmente dólares, euros y yenes) con el fin de prevenir crisis de liquidez entre las economías de la región. La iniciativa Chiang Mai ha evolucionado y ampliado su alcance para convertirse en un mecanismo de seguimiento de los mercados financieros y de prevención y manejo de crisis. En la práctica actúa, además, como un prestamista de último recurso, otorgándole a la región autonomía financiera respecto, por ejemplo, a instituciones como el Fondo Monetario Internacional y el Banco Mundial (Alexander, 2011; Rajan y Rongala, 2008).

La expansión de la integración regional en ASEAN no ha sido sólo hacia los vecinos, sino también interna. Entre 1995 y 1997 ASEAN vivió un proceso de ampliación, incorporando como miembros oficiales a los países de menor desarrollo de la península indochina: Camboya, Laos, Myanmar y Vietnam (CLMV). Estos países presentaban al momento de su incorporación enormes diferencias en cuanto a la estructura económica y social respecto a los miembros originarios del bloque. La incorporación de Myanmar, con un régimen autoritario que ha incurrido en numerosos atentados contra los derechos humanos de su población, ha puesto en apuros a la política regional de ASEAN amenazando la imagen que la región quiere proyectar hacia la comunidad internacional. Lejos de debilitar la integración regional, la incorporación de los CLMV y la crisis financiera asiática ha Ilevado a ASEAN a diseñar un régimen de integración más profundo y ambicioso. En el 2007, los líderes de ASEAN lanzaron el proyecto de la Comunidad Económica de ASEAN (AEC) con los fines de construir un mercado y una estructura de producción única, integrar completamente la región a la economía global, y superar las desigualdades regionales en la región, todo esto con una apretada agenda a ser cumplida el 2015. Como parte del objetivo de superar las desigualdades regionales, $\mathrm{AEC}$ ha reforzado el fondo para el desarrollo regional y la implementación de los Ilamados proyectos NDG (Narrowing Development Gaps) (ASEAN, 2010). Finalmente, el año 2007 se aprobó la Carta Fundamental de ASEAN que representa un fundamental cambio 
institucional para un régimen que se ha caracterizado por la informalidad y flexibilidad de sus procedimientos y reglas. La Carta de ASEAN norma el proceso de generación de AEC, cubriendo aspectos económicos, políticos, de seguridad, sociales y de derechos humanos (Shimizu, 2011; Nesadurai, 2009).

Los cambios institucionales vividos en esta última década en los regímenes regionales de Sudamérica y el sudeste asiático han sido igualmente gatillados por las crisis económicas del capitalismo transnacional, las vulnerabilidades del regionalismo abierto para prevenir y manejar los efectos de las crisis, y la desafección con el sistema internacional financiero. Hay, de hecho, una asombrosa sincronía entre la evolución de las instituciones en ambas regiones del sur global; asimismo, las áreas de desarrollo institucional han sido relativamente similares: integración física, integración energética, mecanismos de redistribución regional, política de superación de asimetrías y desigualdades entre economías, cooperación financiera, protección medioambiental y regulación de mercados productivos (Künhardt, 2007). ¿Cómo interpretar estas transformaciones en tiempos de crisis? ¿Se puede hablar, por ejemplo, del paso de un regionalismo neoliberal a uno posneoliberal? De ser así, ¿cuáles son los contenidos de estos nuevos regímenes de integración regional?

\section{PERSPECTIVAS ACERCA DEL CAMBIO}

En esta sección final quiero enunciar tres perspectivas teóricas acerca del período actual de integración regional. Estas perspectivas comprenden a la vez diagnósticos acerca de la profundidad y relevancia de los cambios institucionales actuales, hipótesis de los posibles escenarios futuros y juicios normativos acerca de lo que debiera esperarse de la integración regional en el sur global, pero también en otras regiones y bajo otros regímenes. La refutación de estas hipótesis depende de la investigación empírica y comparativa de los próximos años

y de las trayectorias de desarrollo de muchas de las políticas e instituciones que han sido creadas en esta década. Por lo mismo, no intento pronunciar un juicio definitivo acerca de estas perspectivas en este ensayo.

\subsection{Perspectiva 1: Posneoliberalismo y arraigamiento regional de los mercados}

Esta perspectiva está basada en una lectura de los procesos de integración regional desde la obra de Karl Polanyi. La obra histórica y teórica de Polanyi estuvo dedicada al estudio de la relación entre economía y vida social, y cómo dicha relación ha variado a lo largo de la historia así como de una sociedad a otra, por ejemplo en el lugar que han ocupado el mercado y el dinero en la organización de la vida social. En la Gran Transformación Polanyi (2001 [1944]) aborda este problema general para el caso de las economías de mercado modernas mostrando cómo en Inglaterra se inicia a partir de finales del siglo XVII un proceso -políticamente conducido- de desarraigo de la actividad económica de la vida social, y la progresiva construcción de mercados autorregulados no solo para todos los tipos de mercancías, sino también para lo que Polanyi denominaría mercancías ficticias: dinero, tierra y trabajo. Estos procesos de mercantilización -que a través del largo siglo XIX se extenderían por el resto de las economías occidentales- generó de modo reactivo respuestas sociales, 
políticas e institucionales con el fin explícito o implícito de des-mercantilizar las esferas de la vida social y proteger tanto a las personas como a la organización social.

La tesis histórica de Polanyi ha sido retomada por economistas políticos como el ya mencionado John Ruggie -en el análisis de los regímenes de dinero y comercio de la posguerra-, Gosta Esping-Andersen -en sus estudios sobre los regímenes de bienestar- y Wolfgang Streeck -en su análisis de los modelos de capitalismo europeos-, entre otros, y elaborada como una teoría del desarrollo capitalista. En efecto, el capitalismo no se ha desarrollado en una trayectoria lineal -como el marxismo vulgar o la teoría de la modernización predecíansino por vaivenes o movimientos dobles, como el mismo Polanyi sostenía. Movimientos de mercantilización y desarraigo han sido seguidos por movimientos de des-mercantilización y re-arraigamiento, en una dialéctica sin término resolutivo entre acumulación capitalista y protección de la reproducción social.

Ha sido el economista político y estudioso de la globalización, Björn Hettne, el primero en utilizar la teoría del desarrollo capitalista de Polanyi en el análisis de los procesos de integración regional. En efecto, el desarrollo de los regímenes de integración regional, como ha sido descrita en la primera sección de este ensayo, puede perfectamente leerse como un doble movimiento. Los regímenes que he denominado políticamente conducidos intentaron anclar la industrialización y la liberalización comercial a objetivos políticos de estabilidad, seguridad y cohesión social. A su vez, los regímenes de integración neoliberales de los ochentas y noventas desanclaron los mercados productivos y de capitales de todo tipo de conducción/intervención sociopolítica. Las crisis financieras de finales de 1990 y de los 2000 serían, desde esta interpretación, consecuencia de dicho proceso de desarraigo y mercantilización a escala global.

Según Hettne (2003), el regionalismo de los 2000 posee características de un rearraigamiento y del retorno de los imperativos políticos por sobre los económicos. De acuerdo a José Antonio Sanahuja, los regímenes que han emergido en Sudamérica en los 2000, fundamentalmente ALBA-TCP (Alianza Bolivariana para los Pueblos de nuestra América-Tratado Comercial de los Pueblos) y UNASUR, corresponderían a regímenes posneoliberales. Ellos expresarían "un retorno de la política a las relaciones exteriores y la política de desarrollo, con una menor atención a la agenda económica y comercial que ha dominado ambas políticas en los últimos años" (Sanahuja, 2011: 120; Motta Veiga y Ríos, 2007).

Siguiendo a Sanahuja, el contenido de los regímenes de integración contemporáneo estaría definido por un retorno del Estado a la política a través de un mayor papel de los actores estatales, frente al protagonismo de los actores privados; un énfasis mayor en la agenda "positiva" de la integración, centrada en la creación de instituciones y políticas comunes y en una cooperación más intensa en ámbitos no comerciales, lo que, como se indicará, ha dado lugar a la ampliación de los mecanismos de cooperación sur-sur, o la aparición de una agenda renovada de paz y seguridad; mayor preocupación por las dimensiones sociales y las asimetrías en cuanto a niveles de desarrollo, y la vinculación entre la integración regional y la reducción de la pobreza y la desigualdad; mayor preocupación por los "cuellos de botella" y las carencias de la infraestructura regional, con el objeto de mejorar la articulación de 
los mercados regionales y, al mismo tiempo, facilitar el acceso a mercados externos; más énfasis en la seguridad energética y la búsqueda de complementariedades en este campo; y finalmente la búsqueda de fórmulas para promover una mayor participación de actores no estatales y la legitimación social de los procesos de integración (Sanahuja, 2011: 121-122).

Esta perspectiva es coincidente con autores como Luiz Carlos Bresser-Pereira, Marcus Kurtz y Sarah Brooks quienes han sostenido la emergencia en regiones de ingreso medio de un "nuevo desarrollismo" (Bresser-Pereira, 2011) o un "neoliberalismo arraigado" (Kurtz y Brooks, 2008), regímenes estado-céntricos, pero no proteccionistas, a través de los cuales las economías emergentes rechazarían las propuestas y reformas de las economías centrales representadas por las instituciones financieras internacionales (véase también Evans, 2009).

\subsection{Perspectiva 2: Capitalismo regulado y mercados regionales inclusivos}

Diversas tradiciones convergen en esta segunda perspectiva. La principal de ellas tiene su origen en los trabajos de David Levi-Faur, Jacint Jordana, John Braithwaite y Peter Draos. De acuerdo a estos autores, las transformaciones del capitalismo post-1980 se pueden entender mejor como la progresiva emergencia de un capitalismo regulado caracterizado por el masivo surgimiento de mercados, regulación pública, regulación privada, híbridos de regulación público/privado, etc. La emergencia de esta regulocracia estaría acompañada por el uso difuso de coordinación social por medio de redes definidas formalmente como conjuntos relativamente estables de relaciones de naturaleza no jerárquicas e interdependiente que vinculan una variedad de actores y organizaciones (Jordana y Levi Faur, 2006), y por la emergencia de variadas formas de gobernanza y metagobernanza (Jessop, 2011; Willke, $2007)^{7}$.

John Braithwaite ha sido enfático en sostener que la interpretación neoliberal del capitalismo, según la cual el rol del Estado estaría desapareciendo, y habría un proceso de desregulación y privatización, es equivocada. No obstante, tampoco sería el caso de un proceso de desmercantilización como se esperaría desde la perspectiva polanyiana. Lo que existen son más y más poderosos mercados con mayor regulación de sus externalidades ejercida por un número creciente y variado de actores públicos y privados, nacionales y transnacionales (Braithwaite, 2008).

Desde esta perspectiva, los regímenes de integración regional no serían sino parte de este proceso de emergencia de estructuras regulativas. De acuerdo a Lászlo Bruszt y Gerald McDermott, los regímenes de integración regionales tienen como propósito la extensión de los mercados y la incorporación de las sociedades nacionales a un ambiente de mercados competitivos; para ello utilizan variados instrumentos con los cuales constituyen y condicionan

Desde esta perspectiva se entiende por gobernanza las estructuras y prácticas involucradas en la coordinación de relaciones sociales complejas, recíprocas e interdependientes, y por metagobernanza la coordinación de tales estructuras y prácticas (Jessop, 2011). La hipótesis de autores sistémicos como Jessop o Willke es que el Estado se diferenciaría progresivamente como un sistema especializado en la metagobernanza de redes y estructuras de autoridad. 
las capacidades de regulación de actores domésticos, sean públicos como privados (Bruszt y McDermott, 2011: 6; véase también Bruszt y Greskovits, 2012, en Revista de Sociología, 26). Instituciones como el Fondo para la Convergencia Estructural del MERCOSUR (FOCEM) o el Fondo de Desarrollo Regional de ASEAN serían precisamente instituciones orientadas a desarrollar capacidades domésticas en aquellas economías con menores recursos: Paraguay y Uruguay en el caso del MERCOSUR y los países CLMV en el caso de ASEAN a través de mecanismos de asistencia técnica, transferencia de tecnología, redistribución de recursos financieros y mejoramiento tecnológico y de las capacidades de gestión. Por otra parte, la Iniciativa Chiang Mai, descrita más arriba, sería una institución orientada a la metagobernanza de los mercados financieros de la región a través del uso de redes de actores múltiples.

Estas instituciones no desmercantilizan los espacios regionales; muy por el contrario ellas extienden los mercados asistiendo, monitoreando y capacitando nuevos actores para su incorporación. Usando los términos de Bruszt y McDermott, los regímenes de integración actúan "como programas de inclusión de actores a los mercados" (Bruszt y Holzhacker, 2009). Desde esta perspectiva, la pregunta de investigación es acerca de la divergencia en los instrumentos utilizados por los distintos regímenes y los distintos grados de influencia sobre las capacidades de los actores domésticos.

Esta perspectiva sustentaría el modelo político que Fernando Henrique Cardoso ha denominado "social democracia global", un modelo que toma en cuenta el poder de los mercados pero los regula, a la vez que incorpora a las masas pobres mediante políticas sociales (Cardoso, 2009). Asimismo hay importantes vínculos con las visiones de instituciones internacionales como el Banco Interamericano de Desarrollo (2011) y el Banco Asiático de Desarrollo (2010).

\subsection{Perspectiva 3: Neoliberalismo y desregulación de los mercados}

Esta perspectiva proviene de los defensores de la tesis de la convergencia esgrimida en la década del noventa (Ohmae, 1994; Gray, 1998), quienes sostenían que los procesos de transnacionalización de los mercados conducirían a una progresiva convergencia de los diversos modelos de capitalismo a un único modelo liberal de mercado.

La lógica de la convergencia supone que la transnacionalización de los mercados conlleva una "carrera al piso" (race to the bottom) en la regulación de salarios, estándares sociales e impuestos a privados. Esto generaría un mecanismo en espiral conducente a nuevas rondas de reformas reduciendo las cargas al capital y asegurando la competitividad de las economías nacionales, reforzado por los mecanismos de cumplimiento de las instituciones financieras internacionales en materias de estabilidad de precios y responsabilidad fiscal (Bohle y Greskovits, 2009).

Llevando este argumento desde el terreno de los capitalismos nacionales a la economía política del regionalismo, Andrew Gamble y Anthony Payne (2003) han argumentado que las instituciones regionales no poseen las capacidades necesarias para regular los procesos de transnacionalización y que, por el contrario, actuarían como adaptaciones 
reactivas ante las externalidades de dichos procesos. John Ravenhill ha argumentado que ASEAN carece de las capacidades institucionales para llevar a cabo el ambicioso proyecto de generar una Comunidad Económica para el 2015. La recuperación de los índices de comercio intrarregional y de flujos de IED poscrisis asiática dicen relación con las operaciones de las empresas transnacionales, más que con los instrumentos de ASEAN (Ravenhill, 2009). Sean Burges, por su parte, ha argumentado que las iniciativas de profundización de la integración en Sudamérica, como IIRSA, se explican por presión de las empresas transnacionales que requieren disminuir los costos de transacción asociados a las dificultades de transporte y comunicación, y a la demanda energética de las economías líderes de la región, fundamentalmente Brasil y su empresa ancla Petrobrás. Según Burges, si bien se suponía que IIRSA dirigiría significativas cantidades de inversión al mejoramiento del sistema de transporte de la región andina, el principal problema continuaría siendo la orientación a materias primas de las empresas transnacionales operando en el continente y la concentración brasileña en el uso de dichas redes transnacionales para la explotación de mercados, sin necesariamente absorber importaciones de sus vecinos. IIRSA ha puesto el énfasis en la creación de rutas de comercio a mercados extrarregionales, sin establecer las sinergias necesarias para la integración de las estructuras productivas que profundizarían la integración económica y política (Burges, 2005).

\section{CONCLUSIONES}

En la introducción al tercer volumen de la Historia Económica de Latinoamérica, Enrique Cárdenas, José Ocampo y Rosemary Thorp sostienen que en los años noventa las palabras proteccionismo, substitución de importaciones e intervencionismo se convirtieron en leyenda negra. Quizá los futuros historiadores escribirán que en el 2020 las palabras desregulación, liberalización y regionalismo abierto se convirtieron también en leyenda negra. No sería de extrañar, de acuerdo a la metáfora del doble movimiento de Polanyi.

Lo cierto es que vivimos un tiempo en el que las crisis asociadas al capitalismo se han hecho más recurrentes en lapsos progresivamente más breves de tiempo. Las economías otrora llamadas centrales se están viendo profundamente golpeadas por dichas crisis, cada una de las cuales comprende uno o varios mercados y circuitos transnacionales. Por su parte, las economías emergentes comienzan a copar no solo los mercados de manufacturas y bienes tecnológicos, sino también los foros desde los cuales se decide y organiza la política internacional.

En este ensayo nos hemos ocupado, desde la economía política y con una perspectiva comparativa, de uno de estos procesos: la integración en regiones en desarrollo y la formación de regímenes de integración. Prácticamente todas las economías emergentes pertenecen a uno de estos regímenes. De los Ilamados países BRICS, por ejemplo, Brasil pertenece al MERCOSUR y a UNASUR manteniendo un papel económico y político protagónico en ambos; China pertenece a ASEAN+3 y Sudáfrica a la Comunidad de Desarrollo Sudafricana (SADC). En cada uno de estos regímenes se han producido interesantes innovaciones institucionales que, sin embargo, están en proceso de implementación. El futuro de la arquitectura de la 
economía global dependerá en buena medida del contenido de los principios y normas que rigen dichas instituciones, así como de la efectividad y desempeño de sus reglas y procedimientos. Los futuros estudios comparativos tendrán que decidir cuál de las hipótesis que en este ensayo hemos bosquejado se ajusta mejor a la realidad de estos regímenes.

\section{BIBLIOGRAFÍA}

Acharya, Amitav (2009): "Arguing about ASEAN: what do we disagree about?", Cambridge Review of International Affairs, 22 (3), pp. 493-499.

ADB (2010): Institutions for Regional Integration. Towards an Asian Economic Community, Asian Development Bank.

Alexander, Jed (2011): "ASEAN-led Regionalism", Flinders Asia Centre Occasional Paper, 4, pp. 1-42.

ASEAN Studies Centre (2008): The ASEAN Community. Unblocking the Roadblocks, Institute of Southeast Asian Studies, Singapore.

ASEAN (2010): Initiative for ASEAN Integration (IAI) Strategic Framework and IAI Work Plan (20092015), en http://www.aseansec.org/22325.pdf

Ba, Alice (2009): "Regionalism's multiple negotiations: ASEAN in East Asia", Cambridge Review of International Affairs, 22 (3), pp. 345-367.

Bhagwati, Jagdish (2004): In defense of Globalization, Oxford University Press.

Balassa, Bela (1973): The Theory of Economic Integration, Allen and Unwin, London.

Beeson, Mark (2009): "ASEAN's way: still fit for purpose?", Cambridge Review of International Affairs, 22 (3), pp. 333-343.

BID (2011): Sector Strategy to Support Competitive Global and Regional Integration, Inter-American Development Bank.

Bohle, Dorothee y Béla Greskovits (2009): "Varieties of Capitalism and Capitalism tout court", European Journal of Sociology, 50, pp. 355-386.

Bowles, Stephen (2004): "Regionalism and development after(?) the global financial crises", en Shaun Breslin, Christopher Hughes, Nicola Phillips y Ben Rosamond (eds.): New Regionalism in the Global Economy, Routledge.

Boyer, Robert (2007): Crisis y regímenes de crecimiento: una introducción a la teoría de la regulación, Miño y Dávila.

Braithwaite, John (2008): Regulatory Capitalism: How it Works, Ideas for Making it Work Better, Edward Elgar Publishing Limited.

Bresser-Pereira, Luiz Carlos (2011): "From Old to New Developmentalism in Latin America", en José Antonio Ocampo y Jaime Ríos (eds.): The Oxford Handbook of Latin American Economics, Oxford University Press.

Bruszt, Lászlo y Béla Greskovits (2012): "Transnacionalización, integración social y diversidades de capitalismo en el este y en el sur", Revista de Sociología, 26.

Bruszt, Lászlo y Ronald Holzhacker (2009): The Transnationalization of Economies, States and Civil Societies. New Challenges for Governance in Europe, Springer. 
Bruszt, Lászlo y Gerald McDermott (2011): “Integrating rule takers: Transnational integration regimes shaping institutional design in emerging market democracies", Review of International Political Economy, pp. 1-37.

Cárdenas, Enrique, José Antonio Ocampo y Rosemary Thorp (2000): An economic history of twentiethcentury Latin America, Palgrave McMillan.

Cardoso, Fernando Henrique (2009): "New Paths: Globalization in Historical Perspective", Studies in Comparative International Development, 44 (4), pp. 269-317.

Cardoso, Fernando Henrique y Enzo Faletto (1969): Dependencia y Desarrollo en América Latina. Ensayo de Interpretación Sociológica, Siglo XXI.

CEPAL (1959): El Mercado Común Latinoamericano (E/CN 12/531), Santiago.

Di Maio, Michel (2011): "Industrial Policies in Developing Countries: History and Perspectives", en Mario Cimoli, Giovanni Dosi y Joseph Stiglitz (eds.): Industrial Policy and Development. The Political Economy of Capabilities Accumulation, Oxford University Press.

Dicken, Peter (2007): Global Shift. Mapping the changing contours of the World Economy, Sage Publications.

Dieter, Heribert (2009): "Changing patterns of regional governance: from security to political economy?", The Pacific Review, 22 (1), pp. 73-90.

Durkheim, Emile (1997) [1893]: The Division of Labour in Society, Free Press.

Evans, Peter (2009): "From Situations of Dependency to Globalized Social Democracy", Studies in Comparative International Development, 44 (4).

Gamble, Andrew y Anthony Payne (2003): "The World Order Approach", en Frederik Söderbaum y Timothy Shaw (eds.): Theories of New Regionalism, Palgrave McMillan.

Gereffi, Gary y Donald Wyman (1990): Manufacturing Miracles: Paths of Industrialization in Latin America and East Asia, Princeton University Press.

Gray, John (1998): False dawn: the delusion of global capitalism, Granta, London.

Haas, Ernst y Philippe Schmitter (1964): “Economics and Differential Patterns of Political Integration: Projections about Unity in Latin America", International Organization, 18 (4), pp. 705-747.

Hasenclever, Andreas, Peter Meyer y Volker Rittberger (1997): Theories of International Regimes, Cambridge University Press.

Hettne, Björn (2003): “The New Regionalism Revisited”, en Frederik Söderbaum y Timothy Shaw (eds.): Theories of New Regionalism, Palgrave McMillan.

INTAL (2006): "Report Mercosur № 11", Inter American Development Bank. (2009a): “Report Mercosur № 13", Inter American Development Bank. (2009b): "Report Mercosur N 14", Inter American Development Bank. (2010): "Report Mercosur N 15", Inter American Development Bank.

Jetschke, Anja (2009): "Institutionalizing ASEAN: celebrating Europe through network governance", Cambridge Review of International Affairs, 22 (3), pp. 407-426.

Jessop, Bob (2011): "Metagovernance", en Mark Bevir (ed.): The SAGE Handbook of Governance, SAGE, London. 
Jordana, Jacint y David Levi Faur (2006): “Towards a Latin American Regulatory State? The Diffusion of Autonomous Regulatory Agencies across Countries and Sectors", International Journal of Public Administration, 29 (4-5), pp. 247-262.

Krasner, Stephen (1982): "Structural Causes and Regime Consequences: regimes as intervening variables", International Organization, 36 (2), pp. 185-205.

Kühnhardt, Ludger (2010): Region-Building. The Global Proliferation of Regional Integration, Berghanh Books.

Kurtz, Marcus y Sarah Brooks (2008): "Embedding Neoliberal Reforms in Latin America", World Politics, 60, pp. 231-280.

Lenz, Tobias (2011): The EU's Inescapable Influence on Global Regionalism, PhD Thesis, University of Oxford.

Lockwood, David (1964): "Social Integration and System Integration", en G.K. Zollschan y H.W. Hirsch (eds.): Exploration in Social Change, Houghton Mifflin, Boston.

Malamud, Andrés (2005): "Mercosur Turns 15: Between Rising Rhetoric and Declining Achievement", Cambridge Review of International Affairs, 18 (3), pp. 421-436.

Malamud, Andrés y Philippe Schmitter (2007): "The Experience of European Integration and the Potential for Integration in South America", IBEI Working Papers, Institut Barcelona d'Estudis Internacionals.

Motta Veiga da, Pedro y Sandra Ríos (2007): O regionalismo pós-liberal, na America do Sul: origem, iniciativas e dilemas, Serie Comercio Internacional, CEPAL.

Nesadurai, Helen (2009): "ASEAN and regional governance after the Cold War: from regional order to regional community?", The Pacific Review, 22 (1), pp. 91-118.

Ocampo, José Antonio (2001): "Raúl Prebisch and the development agenda at the dawn of the twentyfirst century", CEPAL Review, 75, pp. 23-37.

Ohmae, Kenichi (1994): The borderless world: power and strategy in the interlinked economy, Harper Collins, London.

Philips, Nicola (2004): The Southern Cone Model. The political economy of regional capitalist development in Latin America, Routledge/Ripe, London.

Polanyi, Karl (2001) [1944]: The Great Transformation: the political and economic origins of our time, Beacon Press, Boston.

Rajan, Ramkishen y Sunil Rongala (2008): Asia in the Global Economy: finance, trade and investment, World Scientific Publications.

Ravenhill, John (2008): "Fighting irrelevance: an economic community 'with ASEAN characteristics'”, The Pacific Review, 21 (4), pp. 469-488.

Ruggie, John (1982): "International regimes, transactions, and change: embedded liberalism in the postwar economic order", International Organization, 36 (2), pp. 379-415

Sanahuja, José Antonio (2011): "Multilateralismo y regionalismo en clave Suramericana: El caso de UNASUR", Pensamiento Propio, 33, pp. 115-158.

Sbragia, Alberta (2008): "Comparative Regionalism: What might be?", Journal of Common Market Studies, 46, Annual Review, pp. 29-49. 
Shimizu, Kazuchi (2011): "The ASEAN Charter and the ASEAN Economic Community", Economic Journal of Hokkaido University, 40, pp. 73-87.

Stone Sweet, Alec y Wayne Sandholtz (1997): "European Integration and Supranational Governance", Journal of European Public Policy, 4 (3), pp. 297-317.

Streeck, Wolfgang (2009): "Taking Capitalism Seriously Toward an Institutionalist Approach to Contemporary Political Economy", MplfG Discussion Paper, 10/15.

Streeck Wolfgang y Kathleen Thelen (2009): Beyond Continuity: institutional change in advanced political economies, Oxford University Press.

Tèlo, Mario (2007): European Union and New Regionalism: Regional actors and Global Governance in a Post-Hegemonic Era, Ashgate Publishing.

Tussie, Diane (2009): "Latin America: contrasting motivations for regional projects", Review of International Studies, 35, pp. 169-188.

Vázquez, Raúl (2011): "Integración Económica en América Latina: la visión teórica de la CEPAL confrontada con la evolución del proyecto en la región", Journal of Economics, Finance and Administrative Science, 16 (31), pp. 107-118.

Welfens, Paul, Franz Knipping, Suthispand Chirathivat y Cillian Ryan (2006): Integration in Asia and Europe. Historical Dynamics, Political Issues and Economic Perspectives, Springer.

Wiener, Antje y Thomas Diez (2004): European Integration Theory, Oxford University Press.

Willke, Helmut (2007): Smart Governance: governing the Global Knowledge Society, Campus, Frankfurt. 